\title{
Impact and Mitigation of Multiantenna Analog Front-End Mismatch in Transmit Maximum Ratio Combining
}

\author{
Jian Liu, ${ }^{1,2}$ Nadia Khaled, ${ }^{1,3}$ Frederik Petré, ${ }^{1}$ André Bourdoux, ${ }^{1}$ and Alain Barel ${ }^{4}$ \\ ${ }^{1}$ Interuniversity Microelectronics Center (IMEC), Wireless Research, Kapeldreef 75, 3001 Leuven, Belgium \\ ${ }^{2}$ Department ELEC-ETRO, Vrije Universiteit Brussel, 1050 Brussel, Belgium \\ ${ }^{3}$ E.E. Department, KULeuven, ESAT/INSYS, Kapeldreef 75, 3001 Leuven, Belgium \\ ${ }^{4}$ Department of ELEC, Vrije Universiteit Brussel (VUB), Pleinlaan 2, 1050 Brussel, Belgium
}

Received 20 December 2004; Revised 23 May 2005; Accepted 27 May 2005

Transmit maximum ratio combining (MRC) allows to extend the range of wireless local area networks (WLANs) by exploiting spatial diversity and array gains. These gains, however, depend on the availability of the channel state information (CSI). In this perspective, an open-loop approach in time-division-duplex (TDD) systems relies on channel reciprocity between up- and downlink to acquire the CSI. Although the propagation channel can be assumed to be reciprocal, the radio-frequency (RF) transceivers may exhibit amplitude and phase mismatches between the up- and downlink. In this contribution, we present a statistical analysis to assess the impact of these mismatches on the performance of transmit-MRC. Furthermore, we propose a novel mixed-signal calibration scheme to mitigate these mismatches, which allows to reduce the implementation loss to as little as a few tenths of a dB. Finally, we also demonstrate the feasibility of the proposed calibration scheme in a real-time wireless MIMO-OFDM prototyping platform.

Copyright ( 2006 Hindawi Publishing Corporation. All rights reserved.

\section{INTRODUCTION}

High-throughput (HT) wireless local area networks (WLANs) of the fourth-generation, the physical (PHY), and medium access control (MAC) layer of which is currently being standardized in the IEEE 802.11n task group [1] aim to significantly increase the data rate, to significantly improve the quality-of-service (QoS), and to significantly extend the range, compared to existing IEEE $802.11 \mathrm{a} / \mathrm{g}$ type of WLANs. To satisfy these ambitious requirements over the highly space- and frequency-selective indoor propagation channel, multiple-input multiple-output (MIMO) orthogonal frequency-division multiplexing (OFDM) techniques perform low-complexity space-frequency processing to boost the spectral efficiency (and, hence, the data rate), as well as the performance (and, hence, the QoS and/or the range), compared to their single-antenna counterparts [2-4]. In this perspective, transmit maximum ratio combining (TX-MRC) is a simple yet powerful antenna diversity technique that allows to significantly extend the range by exploiting both (transmit) spatial diversity and (transmit) array gain $[5,6]$. It is particularly attractive in multiple-input single-output (MISO) downlink scenarios, where the multiple-antenna access point would optimally weigh the transmit data stream across its antennas, such that channel filtering leads to maximum receive signal-to-noise (SNR) coherent reception at the single-antenna terminal. However, the calculation of the transmit MRC weights requires knowledge of the downlink channel state information (CSI).

For time-division duplexing (TDD) systems, perfect channel reciprocity between up- and downlink is commonly assumed, as long as the round-trip delay is shorter than the channel's coherence time. This assumption allows for an open-loop approach to solve the CSI acquisition problem, in which the CSI estimated during the uplink phase is used for the calculation of the transmit MRC weights employed during the downlink phase. Even though the propagation channel is reciprocal in itself, it has been recently recognised that this is certainly not the case for the radio-frequency (RF) transceivers, which may exhibit significant amplitude and phase mismatches between the up- and downlink as well as across the access point antennas [7-11]. Since these mismatches essentially compromise the correct calculation of the transmit MRC weights, they may result in a severe performance degradation. Hence, there is clearly a need, first, to critically assess the impact of amplitude and phase mismatches on the end-to-end system performance of TX-MRC, and, second, to devise an effective means to mitigate its effect, whenever proven essential and valuable. 


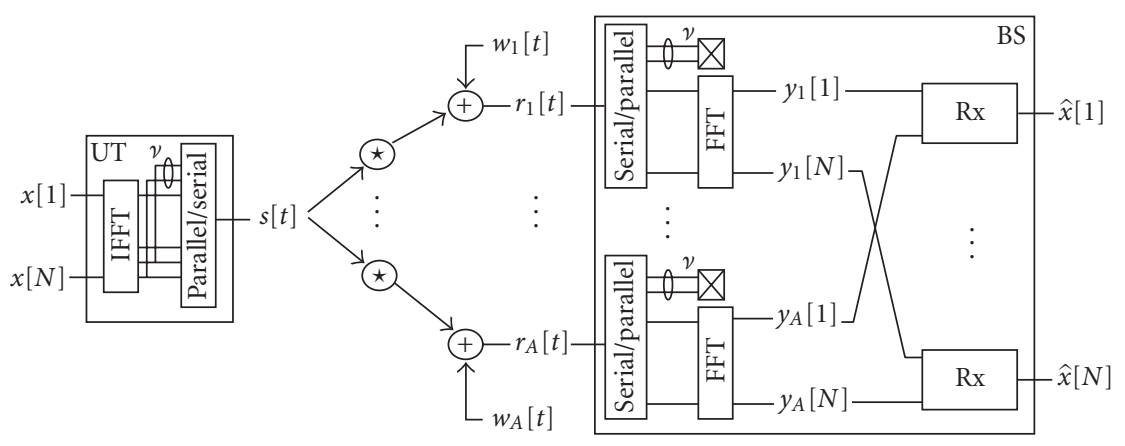

FIgURE 1: The uplink system model.

In this paper, we propose a novel statistical analysis of the impact of multiantenna RF transceivers' amplitude and phase mismatches on transmit MRC. Our analytical approach not only allows both simpler and more reliable evaluation of the impact of each of the mismatches, but, most importantly, it allows to develop a fundamental understanding of their origin and relative importance. In related work, the impact of the mismatches has only been assessed through computer simulations [7-10]. In order to mitigate the multiantenna RF transceivers' problem, we also propose a novel two-step mixed-signal calibration method, which, in a first step, measures, via additional RF calibration hardware, the actual multiantenna transmit and receive front-end mismatches, and, in a second step, compensates for them digitally. Parts of the work described in this paper have been previously published in $[12,13]$.

The paper is organized as follows. Section 2 introduces the system model, including a simple yet realistic model for the multiantenna RF transceivers' amplitude and phase mismatches. Based on this model, Section 3 pursues a statistical approach to assess and evaluate the impact of the different mismatches on the end-to-end performance of transmit MRC. To mitigate the effect of these mismatches, Section 4 describes our mixed-signal calibration method, including its practical implementation and integration in a real-time wireless prototype. Finally, Section 5 summarizes our results and formulates the major conclusions.

\section{Notation}

We use normal letters to represent scalar quantities, boldface lower-case letters to denote column vectors, and boldface upper-case letters to denote matrices. $(\cdot)^{*},(\cdot)^{T}$, and $(\cdot)^{H}$ represent conjugate, transpose, and Hermitian, respectively. Further, $|\cdot|$ and $\|\cdot\|$ represent the absolute value and Frobenius norm, respectively. We reserve $\mathrm{E}\{\cdot\}$ for expectation, and $\lfloor\cdot\rfloor$ for integer flooring. Subscript $a$ points to the ath antenna.

\section{SYSTEM MODEL}

The transmit MRC OFDM-based WLAN communication system, under consideration, is depicted in Figure 1. It consists of an access point (AP) equipped with $A$ antennas and a single-antenna user terminal. In such TDD system, assuming the round-trip delay is shorter than the coherence time of the channel, channel reciprocity is commonly put forth to justify the convenient and spectrally efficient use of the CSI already acquired from the uplink, in the calculation of the transmit MRC weights for the downlink. In this section, we critically evaluate this channel reciprocity assumption. To do that, we accurately model both the uplink channel estimation, which determines the transmit MRC weights, and the downlink data transmission, which actually uses these weights. Our modeling includes the crucial yet commonly neglected contributions of the AP's multiantenna RF transceivers as well as the terminal's single-antenna RF transceiver.

\subsection{Uplink channel estimation}

During the uplink phase, the user terminal groups the incoming data symbols $x[n]$ into blocks of $N$ data symbols. These blocks are denoted by the symbol vector $\mathbf{x}_{m}=$ $\left[x_{m}[1] \cdots x_{m}[N]\right]^{T}$, where $m$ refers to the block index such that $x_{m}[n]=x[m N+n]$. Each symbol vector $\mathbf{x}_{m}$ is fed into an $N$-tap IFFT to generate the time-domain sequence $\mathbf{s}_{m}$. A cyclic prefix of length $\nu$ is prepended to this sequence, which is then converted to a serial stream. The resulting sequence $\left[s_{m}[N-v] \cdots s_{m}[N] s_{m}[1] \cdots s_{m}[N]\right]$ is digital-toanalog converted. The continuous-time signal $s(t)$ is sent through the $A$ convolutional channels $f_{R x, a}(t) \star h_{a}(t) \star g_{T x}(t)$, which each represents the concatenation of the equivalent baseband representations of the terminal's transmit frontend $g_{T x}(t)$, the multipath propagation channel $h_{a}(t)$, and the receive front-end $f_{R x, a}(t)$ corresponding to the AP's ath antenna. At the output of the AP's ath receive front end, the received time domain signal $r_{a}(t)$, which consists of the convolutional product $f_{R x, a}(t) \star h_{a}(t) \star g_{T x}(t) \star s(t)$ and an AWGN term $f_{R x, a}(t) \star w_{a}(t)$, is converted to the digital domain and again grouped into blocks of size $N+\nu$. After discarding the $\nu$-sample cyclic prefix and taking the $N$-tap FFT of each received block, we end up with $A$ received sequences $y_{a}[n]$ on each carrier. These signals $y_{a}[n]$ are then postprocessed to estimate the frequency-domain counterparts of the $A$ convolutional channels $f_{R x, a}(t) \star h_{a}(t) \star g_{T x}(t)$, and subsequently to provide estimates $\hat{x}_{m}[n]$ for the transmitted symbols $x_{m}[n]$.

If $v$ is larger than the length of composite channels $f_{R x, a}(t) \star h_{a}(t) \star g_{T x}(t)$, the linear convolution is observed as 


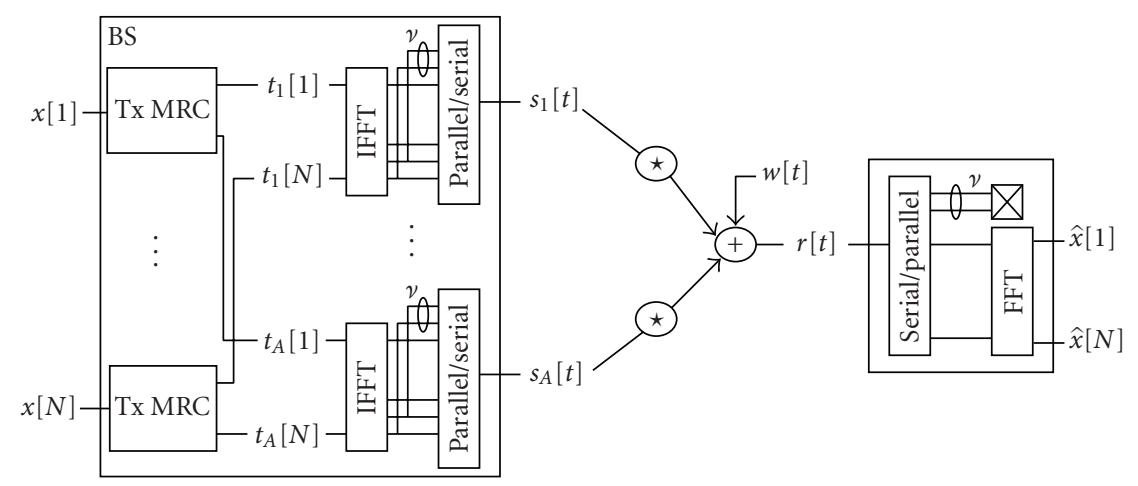

FIgure 2: The downlink transmit MRC system model.

cyclic by the AP. Thus, in the frequency domain, it becomes equivalent to multiplication with the discrete Fourier transform of the composite channel, given by $f_{R x, a}[n] \cdot h_{a}[n]$. $g_{T x}[n]$. Consequently, the data model on subcarrier $n$ reads:

$$
\begin{aligned}
& {\left[\begin{array}{c}
y_{1}[n] \\
\vdots \\
y_{A}[n]
\end{array}\right]=} \underbrace{\left[\begin{array}{c}
f_{R x, 1}[n] \cdot h_{1}[n] \\
\vdots \\
f_{R x, A}[n] \cdot h_{A}[n]
\end{array}\right] \cdot g_{T x}[n] \cdot x[n]}_{\operatorname{CSI}_{\text {uplink }}[n]} \\
&+\left[\begin{array}{c}
f_{R x, 1}[n] \cdot w_{1}[n] \\
\vdots \\
f_{R x, A}[n] \cdot w_{A}[n]
\end{array}\right]
\end{aligned}
$$

where the OFDM symbol block index $m$ has been dropped because we are interested in block-by-block detection of $\mathbf{x}_{m}$. Clearly, OFDM modulation decouples the convolutional composite channel into a set of $N$ orthogonal flat-fading composite channels, on the $N$ subcarriers. This property is exploited by the AP to carry out data detection on each subcarrier independently. Accordingly, on subcarrier $n, \hat{x}[n]$ is detected based on the estimated frequency-domain composite channel $\mathbf{C S I}_{\text {uplink }}[n]$ on that subcarrier. ${ }^{1}$

\subsection{Downlink data communication}

Before the actual data communication can start, the AP computes the transmit MRC precoder $\mathbf{p}[n]$ corresponding to each subcarrier $n$, based on the previously acquired $\mathrm{CSI}_{\text {uplink }}[n]$. This $A \times 1$-dimensional transmit MRC precoder is defined as

$$
\mathbf{p}[n]=\frac{\operatorname{CSI}_{\text {uplink }}[n]^{H}}{\left\|\operatorname{CSI}_{\text {uplink }}[n]\right\|_{2}} .
$$

During the downlink phase, the transmit MRC precoder $\mathbf{p}[n]$ is then used, on subcarrier $n$, to spatially spread the in-

\footnotetext{
${ }^{1}$ We assume perfect uplink channel estimation to be able to isolate and assess the impact of the RF transceivers mismatches on the system's performance.
}

put symbol $x[n]$ into $A$ symbols $\mathbf{t}[n]=\left[t_{1}[n] \cdots t_{A}[n]\right]^{T}$, to be transmitted on the $A$ transmit antennas as shown in Figure 2. On each antenna $a$, the transmit symbols $t_{a}[n]$ are subsequently grouped into blocks of $N$ symbols and converted to the time-domain sequence $\mathbf{s}_{a}(t)$ via an $N$-tap IFFT. A cyclic prefix of length $v$ is inserted into the sequence, which is then serialized. Each resulting transmit stream $\left[s_{a}[N-v] \cdots s_{a}[N] s_{a}[1] \cdots s_{a}[N]\right]$ is digital-to-analog converted and launched into the convolutional channel $g_{R x}(t) \star$ $h_{a}(t) \star f_{T x, a}(t)$, which now represents the concatenation of the equivalent baseband representations of the transmit front-end $f_{T x, a}(t)$ and the multipath propagation channel $h_{a}(t)$, corresponding to the AP's ath antenna, and the terminal's receive front-end $g_{R x}(t)$. At the output of this receive front end, the terminal receives the convolutional mixture $r(t)=g_{R x}(t) \star \sum_{a=1}^{A} h_{a}(t) \star f_{T x, a}(t) \star s_{a}(t)$ and an AWGN term $g_{R x}(t) \star w(t)$. Subsequently, it digitizes $r(t)$, removes the cyclic prefix, and takes the $N$-tap FFT. The resulting frequency domain received symbol $\hat{x}[n]$ represents an estimate for $x[n]$, and can easily seen to be

$$
\begin{aligned}
\hat{x}[n]= & \underbrace{g_{R x}[n] \cdot\left[h_{1}[n] \cdot f_{T x, 1}[n] \cdots h_{A}[n] \cdot f_{T x, A}[n]\right]}_{\operatorname{CSI}_{\mathrm{downlink}}[n]} \\
& \cdot \mathbf{p}[n] \cdot x[n]+g_{R x}[n] \cdot w[n] .
\end{aligned}
$$

Replacing $\mathbf{p}[n]$ by its expression of (2), where $\mathbf{C S I}_{\text {uplink }}$ is given by (1), the received signal of (3) can be explicitly rewritten:

$$
\hat{x}=\underbrace{\frac{g_{R x} g_{T x}^{*}}{\left|g_{T x}\right|}}_{\text {user terminal related }} \cdot \frac{\sum_{a=1}^{A}\left|h_{a}\right|^{2} f_{T x, a} f_{R x, a}^{*}}{\sqrt{\sum_{a=1}^{A}\left|h_{a} f_{R x, a}\right|^{2}}} \cdot x+g_{R x} \cdot w,
$$

where the subcarrier $n$ is dropped for notational brevity. Nevertheless, all equations and results of this section are formulated and should be understood per subcarrier. Furthermore, the receive SNR, per subcarrier, is given by

$$
\mathrm{SNR}=\frac{\left.\left.\left|\sum_{a=1}^{A}\right| h_{a}\right|^{2} f_{T x, a} f_{R x, a}^{*}\right|^{2}}{\sum_{a=1}^{A}\left|h_{a} f_{R x, a}\right|^{2}} \cdot \frac{E_{s}}{\sigma_{w}^{2}},
$$


where $E_{s} / \sigma_{w}^{2}$ is the average transmit power over the receiver noise power. It would also correspond to the average receive SNR for a single-input single-output (SISO) system with the same average transmit power. The receive SNR of (5) exclusively determines the performance of the transmit MRC system. Clearly, the user terminal related coefficient in (4) does not alter the performance of the transmit MRC. Consequently, the user terminal front end will be omitted in the subsequent analysis. Moreover, (5) shows that the amplitude and phase mismatches in the multiantenna AP transceivers disturb the response of the transmit MRC. Indeed, the ideal response is given by $[5,6]$ :

$$
\mathrm{SNR}_{\text {ideal }}=\sum_{a=1}^{A}\left|h_{a}\right|^{2} \cdot \frac{E_{s}}{\sigma_{w}^{2}} .
$$

In order to quantify the nature and magnitude of the disturbance caused by amplitude and phase mismatches, in the AP's multiantenna RF transceivers, a model of these nondeterministic and time-varying mismatches needs to be formulated.

\subsection{Amplitude and phase mismatch model}

An ideal front end has a baseband equivalent response of unit amplitude and zero phase. Because of random process variations, an actual front end will exhibit a random response around this nominal ideal response. The magnitude of the exhibited deviation from the nominal response depends on the magnitude of process variations. In all the following, we equivalently refer to the random front-end response, around the ideal one, as a mismatch in the front-end response.

On a given subcarrier, we model the mismatches in the responses of the transmit and receive front ends using complex gains $f=|f| e^{j \arg (f)}$, where $|f|$ and $e^{j \arg (f)}$ represent the amplitude and phase mismatches, respectively. Moreover, we consider only a first-order approximation of the front-end behavior such that nonlinearities can be neglected. Under this approximation, $|f|$ and $e^{j \arg (f)}$ as well as their stochastic distributions can be considered independent. The latter stochastic distributions are as follows:

(i) the amplitude $|f|$ is modeled as a real Gaussian variable. Its mean value is here given by the unit nominal ideal value and its variance is denoted $\sigma_{\| \mid}^{2}$. While the Gaussian model is commonly used to model RF amplitude errors, it is assumed that the variance $\sigma_{\|}^{2}$ is small, up to $40 \%$, such that the occurrence of negative realizations is negligible,

(ii) the angle $\arg (f)$ is considered to be uniformly distributed in the range $[-\Phi, \Phi]$. The uniform distribution was retained as a worst-case distribution due to the large variability of the phase as well as the inherent ambiguity of its baseband equivalent representation, defined only between $[-\pi, \pi[$.

The parameters $\sigma^{2}$ and $\Phi$ reflect how well the branches of the multiantenna transmit/receive front end are matched. These parameters may be different for the transmit and receive paths.

\subsection{Practical system parameters}

When quantifying the impact of the amplitude and phase mismatches, in the AP's multiantenna RF transceivers, on transmit MRC, an AP with $A=2$ antennas will be instantiated. The OFDM-based IEEE 802.11a indoor WLAN standard [14] will be used for all physical layer parameters. For instance, there are $N=64$ subcarriers of which 48 are used for data and a $v=16$-sample cyclic prefix. Furthermore, the proposed IEEE 802.11 TGn channel D [15], which models typical indoor channels with a 50 ns rms delay spread, will be used for all Monte Carlo performance simulations. Finally, we consider amplitude mismatches up to $\sigma_{\|}=40 \%$, and phase mismatches corresponding to up to $\Phi=180 \mathrm{de}-$ grees.

\section{IMPACT ANALYSIS}

To gain insight into their respective contributions to the degradation of the system performance, we evaluate the impact of each mismatch separately. The performance is measured in terms of the SNR loss with respect to the ideal transmit MRC response, $R=\mathrm{SNR} / \mathrm{SNR}_{\text {ideal }}$. Note that $R$ is expressed in linear units.

\subsection{Transmit amplitude mismatch only}

This scenario arises when the $A$ receive front-end chains are ideal and the $A$ transmit front-end phases are equal to zero:

$$
\begin{aligned}
\left\{f_{R x, a}\right\}_{1 \leq a \leq A} & =1, \\
\left\{\arg \left(f_{T x, a}\right)\right\}_{1 \leq a \leq A} & =0 .
\end{aligned}
$$

The resulting SNR loss, $R$ is given by

$$
R=\left(\frac{\sum_{a=1}^{A}\left|h_{a}\right|^{2}\left|f_{T x, a}\right|}{\sum_{a=1}^{A}\left|h_{a}\right|^{2}}\right)^{2}
$$

Based on the mismatch model introduced in Section 2.3, the transmit amplitude mismatches $\left\{\left|f_{T x, a}\right|\right\}_{a}$ are independent identically distributed (i.i.d) Gaussian variables of unit mean and variance $\sigma_{\|}^{2}$, that is, $\left|f_{T x, a}\right| \sim \mathcal{N}\left(1, \sigma_{\| \mid}^{2}\right)$. This model, however, would artificially lead to an increase of the average transmit power by a factor $\left(1+\sigma_{\| \mid}^{2}\right)$, which is basically the mean of $\left|f_{T x, a}\right|^{2}$. Consequently, the transmit amplitude mismatches must be normalized to ensure that the average transmit power is $E_{s}$. Thus, the transmit amplitude mismatch should rather be modeled as

$$
\left|f_{T x}\right| \sim \mathcal{N}\left(\frac{1}{\sqrt{1+\sigma_{\|}^{2}}}, \frac{\sigma_{\|}^{2}}{\left(1+\sigma_{\|}^{2}\right)}\right) .
$$

Being a sum of scaled versions of independent Gaussian variables, the numerator of $(8)$ is also Gaussian distributed as $\mathcal{N}\left(\left(\sum_{a=1}^{A}\left|h_{a}\right|^{2}\right) / \sqrt{1+\sigma_{\|}^{2}},\left(\sum_{a=1}^{A}\left|h_{a}\right|^{4}\right) \sigma_{\|}^{2} /\left(1+\sigma_{\|}^{2}\right)\right)$. The division by the denominator, $\sum_{a=1}^{A}\left|h_{a}\right|^{2}$, leads to a ratio that is 


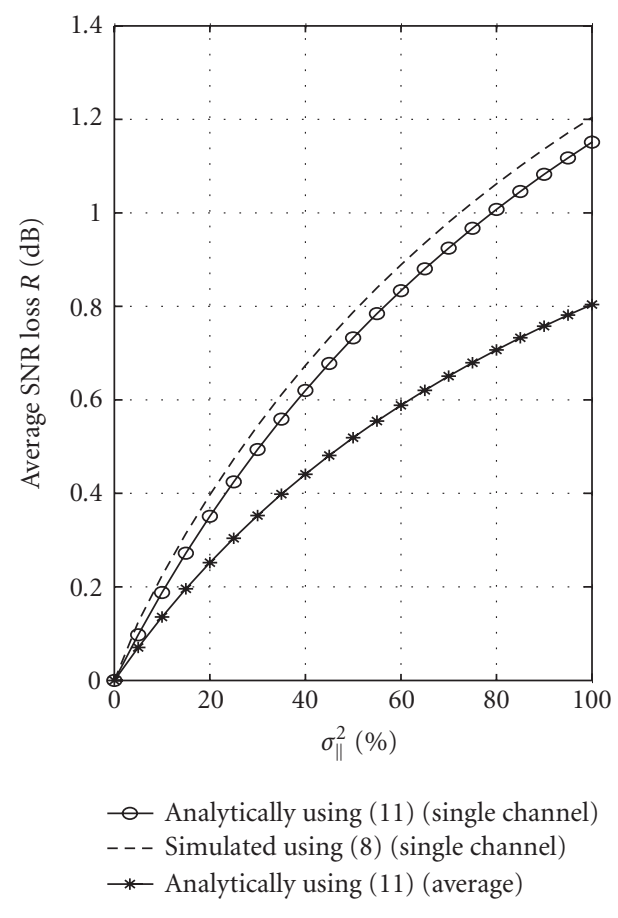

(a)

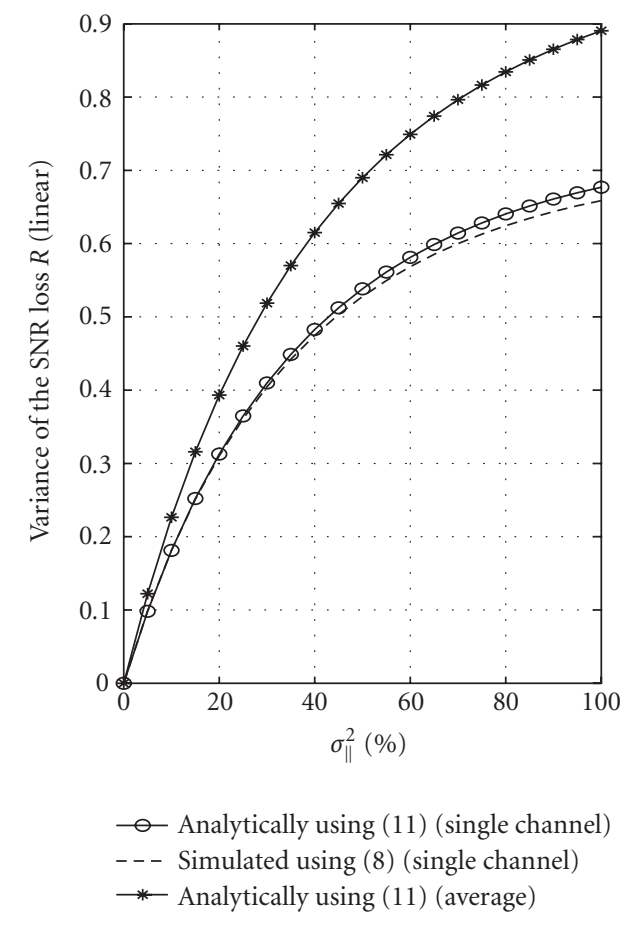

(b)

FIgURE 3: The average (in $\mathrm{dB}$ ) and the variance (in linear units) of the SNR loss $\mathrm{R}$ in presence of transmit amplitude mismatches, for $A=2$ transmit antennas.

Gaussian distributed:

$$
\sqrt{R} \sim \mathcal{N}\left(\mu=\frac{1}{\sqrt{1+\sigma_{\|}^{2}}}, \sigma^{2}=\frac{\sigma_{\|}^{2}}{1+\sigma_{\|}^{2}} \frac{\sum_{a=1}^{A}\left|h_{a}\right|^{4}}{\left(\sum_{a=1}^{A}\left|h_{a}\right|^{2}\right)^{2}}\right) .
$$

Finally, as the square of a noncentral Gaussian variable, $R$ follows a noncentral Chi-square distribution [16] with mean $E\{R\}=\mu^{2}+\sigma^{2}$ and variance $\operatorname{Var}\{R\}=4 \mu^{2} \sigma^{2}+2 \sigma^{4}$ :

$$
R \sim \mathcal{X}_{1,1}\left(\mu^{2}+\sigma^{2}, 4 \mu^{2} \sigma^{2}+2 \sigma^{4}\right) .
$$

Interestingly, the mean of the linear SNR loss, $\mathrm{R}$, reads:

$$
E\{R\}=\frac{1}{1+\sigma_{\|}^{2}}\left(1+\sigma_{\|}^{2} \frac{\sum_{a=1}^{A}\left|h_{a}\right|^{4}}{\left(\sum_{a=1}^{A}\left|h_{a}\right|^{2}\right)^{2}}\right) \leq 1 .
$$

The aim of the proposed statistical analysis is to provide an accurate characterization of the SNR degradation induced by the multiantenna RF transceivers' transmit amplitude mismatches on the performance of transmit MRC. Figure 3 validates this analysis and quantifies the resulting SNR degradation for the practical transmit MRC system described in Section 2.4 .

\subsection{Transmit phase mismatch only}

This scenario occurs when the $A$ receive front-end chains are ideal, and the $A$ transmit front-end amplitudes are equal to one:

$$
\begin{aligned}
\left\{f_{R x, a}\right\}_{1 \leq a \leq A} & =1, \\
\left\{\left|f_{T x, a}\right|\right\}_{1 \leq a \leq A} & =1 .
\end{aligned}
$$

The corresponding linear SNR loss, $R$, reads:

$$
R=\left|\frac{\sum_{a=1}^{A}\left|h_{a}\right|^{2} e^{j \arg \left(f_{T x, a}\right)}}{\sum_{a=1}^{A}\left|h_{a}\right|^{2}}\right|^{2}
$$

To identify the statistics of $R$, we substitute $e^{j \arg \left(f_{T x, a}\right)}=$ $\cos \left[\arg \left(f_{T x, a}\right)\right]+j \sin \left[\arg \left(f_{T x, a}\right)\right]$ and develop (14) into

$$
R=1+\frac{2 \sum_{i<j}\left|h_{i} h_{j}\right|^{2}}{\left(\sum_{a=1}^{A}\left|h_{a}\right|^{2}\right)^{2}}\left(Y_{i, j}-1\right)
$$

where $Y_{i, j}=\cos \left[\arg \left(f_{T x, i}\right)-\arg \left(f_{T x, j}\right)\right]$. We further introduce the set of random variables $\left\{Z_{i}=\cos \left[\arg \left(f_{T x, i}\right)\right]\right\}_{1 \leq i \leq A}$. This choice is motivated by the fact that the joint distribution of $\left\{Z_{i}\right\}_{i}$, contrarily to that of $\left\{Y_{i, j}\right\}_{i, j}$, is easily related to that of $\left\{\arg \left(f_{T x, i}\right)\right\}_{i}$, as follows:

$$
f_{\{Z\}_{i}}\left(z_{1}, \ldots, z_{A}\right)=\frac{1}{\Phi^{A}} \frac{1}{\prod_{i=1}^{A} \sqrt{1-z_{i}^{2}}}, \quad\left\{z_{i}\right\}_{i} \in[\cos \Phi, 1]
$$


The desired variable $Y_{i, j}$ can be rewritten in terms of the new variables $\left\{Z_{i}\right\}_{i}$ :

$$
Y_{i, j}=\left\{\begin{array}{l}
Z_{i} Z_{j}+\sqrt{1-Z_{i}^{2}} \sqrt{1-Z_{j}^{2}}, \\
\operatorname{sign}\left[\arg \left(f_{T x, i}\right)\right]=\operatorname{sign}\left[\arg \left(f_{T x, j}\right)\right], \\
Z_{i} Z_{j}-\sqrt{1-Z_{i}^{2}} \sqrt{1-Z_{j}^{2}}, \\
\operatorname{sign}\left[\arg \left(f_{T x, i}\right)\right] \neq \operatorname{sign}\left[\arg \left(f_{T x, j}\right)\right] .
\end{array}\right.
$$

Based on (16) and (17), the expected value $E\left\{Y_{i, j}\right\}$ is easily found to be

$$
E\left\{Y_{i, j}\right\}=\frac{\sin ^{2} \Phi}{\Phi^{2}}
$$

Consequently, the expected value of the SNR loss $R$, in (15) can then be drawn:

$$
E\{R\}=1+\frac{\left(\sum_{a=1}^{A}\left|h_{a}\right|^{2}\right)^{2}-\sum_{a=1}^{A}\left|h_{a}\right|^{4}}{\left(\sum_{a=1}^{A}\left|h_{a}\right|^{2}\right)^{2}}\left[\frac{\sin ^{2} \Phi}{\Phi^{2}}-1\right] .
$$

We now similarly determine the variance of $R, \operatorname{Var}\{R\}$, given by

$$
\operatorname{Var}\{R\}=\operatorname{Var}\{1+\sum_{i<j} \underbrace{\frac{2\left|h_{i} h_{j}\right|^{2}}{\left(\sum_{a=1}^{A}\left|h_{a}\right|^{2}\right)^{2}}}_{\alpha_{i, j}}\left(Y_{i, j}-1\right)\} .
$$

Since $\left\{Y_{i, j}\right\}_{i, j, i<j}$ are identically distributed yet statistically dependent, the previous expression develops into

$$
\begin{aligned}
\operatorname{Var}\{R\}= & \sum_{i<j} \alpha_{i, j}^{2} \operatorname{Var}\left\{Y_{i, j}\right\} \\
& +\sum_{\{i, j\} \neq\{k, l\} \&(i<j) \&(k<l)} \alpha_{i, j} \alpha_{k, l}\left(E\left\{Y_{i, j} Y_{k, l}\right\}\right. \\
& \left.-E\left\{Y_{i, j}\right\} E\left\{Y_{k, l}\right\}\right) .
\end{aligned}
$$

The evaluation of $\operatorname{Var}\left\{Y_{i, j}\right\}$, based on (16) and (17), can be shown to lead to

$$
\operatorname{Var}\left\{Y_{i, j}\right\}=\frac{1}{2}\left(\frac{\sin ^{2}[2 \Phi]}{(2 \Phi)^{2}}+1\right)-\frac{\sin ^{4}[\Phi]}{\Phi^{4}} .
$$

Recalling the previously calculated expectation $E\left\{Y_{i, j}\right\}$ used in (18), we only need to identify the correlation $E\left\{Y_{i, j} Y_{k, l}\right\}$ for $(\{i, j\} \neq\{k, l\}) \&(i<j) \&(k<l)$. Due to the conditional expression $Y_{i, j}$, the various correlations $E\left\{Y_{i, j} Y_{k, l}\right\}$ can only be evaluated in closed form for a given $A$. For illustration, we propose their expressions as well as the corresponding $\operatorname{Var}\{R\}$ expressions, for $A=\{2,4\}$ transmit antennas. For the simple case, where the AP has only $A=2$ antennas, it is clear that the expression in (21) reduces to

$$
\operatorname{Var}\{R\}=\alpha_{1,2}^{2} \operatorname{Var}\left\{Y_{1,2}\right\}
$$

Replacing $\operatorname{Var}\left\{Y_{1,2}\right\}$ by its explicit expression of (22), the variance of $R$, for $A=2$, is given by

$$
\begin{aligned}
\operatorname{Var}\{R\}= & 4\left[\frac{1}{2}\left(\frac{\sin ^{2}[2 \Phi]}{(2 \Phi)^{2}}+1\right)-\frac{\sin ^{4}[\Phi]}{\Phi^{4}}\right] \\
& \times \frac{\left|h_{1}\right|^{4}\left|h_{2}\right|^{4}}{\left(\left|h_{1}\right|^{2}+\left|h_{2}\right|^{2}\right)^{4}} .
\end{aligned}
$$

When the AP has $A=4$ antennas, we need to evaluate the expectation $E\left\{Y_{i, j} Y_{k, l}\right\}$ for $(\{i, j\} \neq\{k, l\}) \&(i<j) \&(k<l)$. The resulting two expressions are:

$$
\begin{gathered}
E\left\{Y_{i, j} Y_{i, l}\right\}_{j \neq l}=\frac{(\Phi+\sin [\Phi] \cos [\Phi]) \sin ^{2}[\Phi]}{2 \Phi^{3}}, \\
E\left\{Y_{i, j} Y_{k, l}\right\}_{(i \neq k) \&(j \neq l)}=\frac{\sin ^{4}[\Phi]}{\Phi^{4}}
\end{gathered}
$$

Based on the results in (22) and (25), the variance of $R$, in presence of transmit phase mismatch can be reduced to

$$
\begin{aligned}
\operatorname{Var}\{R\} & =4\left[\frac{1}{2}\left(\frac{\sin ^{2}[2 \Phi]}{(2 \Phi)^{2}}+1\right)-\frac{\sin ^{4}[\Phi]}{\Phi^{4}}\right] \frac{\sum_{i<j}\left|h_{i}\right|^{4}\left|h_{j}\right|^{4}}{\left(\sum_{a=1}^{4}\left|h_{a}\right|^{2}\right)^{4}} \\
& \times 4\left[\frac{(\Phi+\sin [\Phi] \cos [\Phi]) \sin ^{2}[\Phi]}{2 \Phi^{3}}-\frac{\sin ^{4}[\Phi]}{\Phi^{4}}\right] \\
& \cdot \frac{\left(\sum_{i<j}\left|h_{i} h_{j}\right|^{2}\right)^{2}-\sum_{i<j}\left|h_{i} h_{j}\right|^{4}-6\left|h_{1} h_{2} h_{3} h_{4}\right|^{2}}{\left(\sum_{a=1}^{4}\left|h_{a}\right|^{2}\right)^{4}} .
\end{aligned}
$$

Even though, we only evaluated $\operatorname{Var}\{R\}$ of (21) for $A=$ $\{2,4\}$, which are of interest in this work. The same proposed approach can be used to evaluate the variance of the linear SNR loss, provided the adaptation of the joint probability density function $f_{\{Z\}_{i}}\left(z_{1}, \ldots, z_{A}\right)$ and a careful count of all the involved cross terms $E\left\{Y_{i, j} Y_{k, l}\right\}$.

Figure 4 validates the analytically calculated mean and variance of SNR loss $R$, as it shows that they perfectly fit the simulated ones for the practical system previously introduced in Section 2.4.

\subsection{Receive amplitude mismatch only}

This scenario depicts the case when the $A$ transmit RF chains are ideal, and the $A$ receive front-end phases are equal to zero:

$$
\begin{aligned}
\left\{f_{T x, a}\right\}_{1 \leq a \leq A} & =1, \\
\left\{\arg \left(f_{R x, a}\right)\right\}_{1 \leq a \leq A} & =0 .
\end{aligned}
$$

The linear SNR loss, $R$, is now expressed as

$$
R=\frac{1}{\sum_{a=1}^{A}\left|h_{a}\right|^{2}}\left(\sum_{a=1}^{A}\left|h_{a}\right| \frac{\left|h_{a}\right|\left|f_{R x, a}\right|}{\sqrt{\sum_{a=1}^{A}\left|h_{a} f_{R x, a}\right|^{2}}}\right)^{2} .
$$




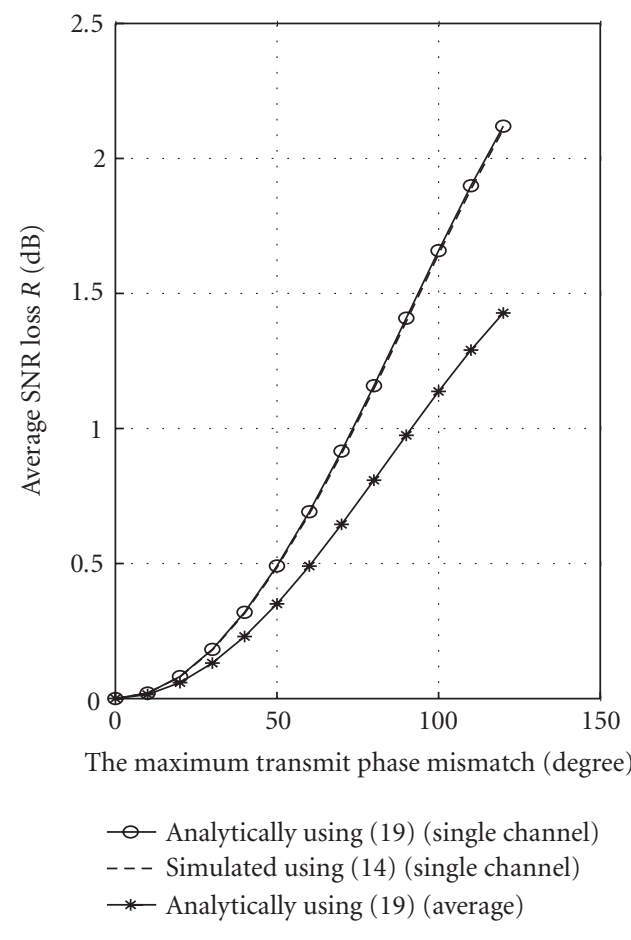

(a)

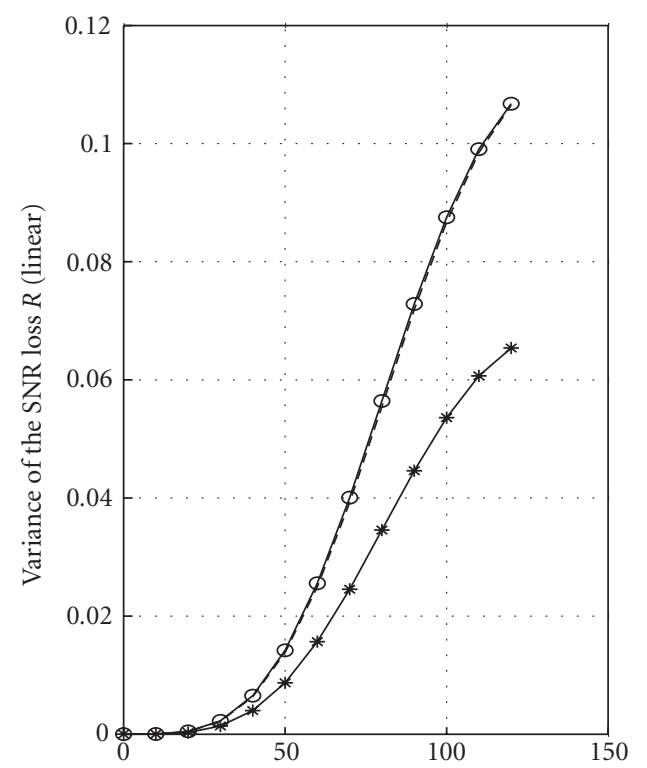

The maximum transmit phase mismatch (degree)

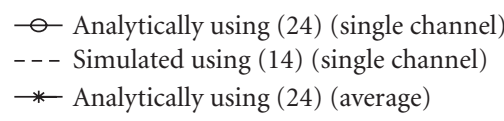

(b)

FIgURe 4: The average (in $\mathrm{dB}$ ) and the variance (in linear units) of the SNR loss $\mathrm{R}$ in presence of transmit phase mismatches, for $A=2$ transmit antennas.

We note that each $x_{a}=\left|h_{a}\right|\left|f_{R x, a}\right|$ is Gaussian distributed as $\mathcal{N}\left(\mu_{a}=\left|h_{a}\right|, \operatorname{var}_{a}=\sigma_{\|}^{2}\left|h_{a}\right|^{2}\right)$. Furthermore, $\left\{x_{a}\right\}_{1 \leq a \leq A}$ are statistically independent. Thus, their joint probability density function (pdf) is simply given by

$$
p\left(x_{1}, \ldots, x_{A}\right)=\frac{1}{(\sqrt{2 \pi})^{A} \sqrt{\prod_{a=1}^{A} \operatorname{var}_{a}}} e^{-\sum_{a=1}^{A}\left(\left(x_{a}-\mu_{a}\right)^{2} / 2 \operatorname{var}_{a}\right)} .
$$

The mean as well as the variance of $R$ can then be determined by evaluating two $M_{T}$-tuple infinite integrals over $\left\{x_{a}\right\}_{1 \leq a \leq A}$.

$$
\begin{gathered}
E\{R\}=\int_{-\infty}^{+\infty} \cdots \int_{-\infty}^{+\infty} R \cdot p\left(x_{1}, \ldots, x_{A}\right) d x_{1} \cdots d x_{A}, \\
\operatorname{Var}\{R\}=\int_{-\infty}^{+\infty} \cdots \int_{-\infty}^{+\infty} R^{2} \cdot p\left(x_{1}, \ldots, x_{A}\right) d x_{1} \cdots d x_{A} \\
\quad-E^{2}\{R\},
\end{gathered}
$$

where $R$ of (28) is rewritten as

$$
R=\frac{1}{\sum_{a=1}^{A}\left|h_{a}\right|^{2}} \cdot\left(\left[\left|h_{1}\right| \cdots\left|h_{A}\right|\right]\left(\begin{array}{c}
x_{1} \\
\vdots \\
x_{A}
\end{array}\right) \frac{1}{\sqrt{\sum_{a=1}^{A} x_{a}^{2}}}\right)^{2} .
$$

To ensure both the convergence and ease of the numerical integration, we try to convert both infinite integrals
(30) to finite ones. This is achieved by making the simple but key observation that the $A$-dimensional vector $Y=$ $\left[x_{1} \cdots x_{A}\right]^{T} / \sqrt{\sum_{a=1}^{A} x_{a}^{2}}$ lies on the $A$-dimensional unit hypersphere. Consequently, it can be represented using the $A$ dimensional spherical coordinates $\left(r, \phi_{1}, \ldots, \phi_{A-1}\right)$, whose pdf can be simply related to that of $\left\{x_{a}\right\}_{1 \leq a \leq A}$, as follows:

$$
\begin{gathered}
p\left(r, \phi_{1}, \ldots, \phi_{A-1}\right)=r^{A-1} \prod_{a=2}^{A} \sin ^{A-a} \phi_{A-a+1} \cdot p\left(x_{1}, \ldots, x_{A}\right), \\
r \in[0,+\infty), \phi_{1} \in[0,2 \pi],\left\{\phi_{a}\right\}_{2 \leq a \leq A-1} \in[0, \pi] .
\end{gathered}
$$

Straightforward developments of (32) lead to

$$
\begin{aligned}
& p\left(r, \phi_{1}, \ldots, \phi_{A-1}\right)=c \cdot r^{A-1} \cdot e^{-a r^{2}+b r}, \\
& \quad r \in[0,+\infty), \phi_{1} \in[0,2 \pi],\left\{\phi_{a}\right\}_{2 \leq a \leq A-1} \in[0, \pi],
\end{aligned}
$$

where $a, b$, and $c$ are given by

$$
\begin{aligned}
& a=\frac{\cos ^{2} \phi_{A-1}}{2 \operatorname{var}_{A}}+\sum_{a=1}^{A-1} \frac{\prod_{k=1}^{A-1} \sin ^{2} \phi_{k} \cos \phi_{a-1}}{2 \operatorname{var}_{a}}, \\
& b=\frac{\mu_{A} \cos \phi_{A-1}}{\operatorname{var}_{A}}+\sum_{a=1}^{A-1} \frac{\mu_{a} \prod_{k=1}^{A-1} \sin \phi_{k} \cos \phi_{a-1}}{\operatorname{var}_{a}}, \\
& c=\frac{\prod_{a=2}^{A} \sin ^{A-a} \phi_{A-a+1}}{(\sqrt{2 \pi})^{A} \prod_{a=1}^{A} \sqrt{\operatorname{var}_{a}}} \cdot e^{-\sum_{a=1}^{A}\left(\mu_{a}^{2} / 2 \operatorname{var}_{a}\right)},
\end{aligned}
$$




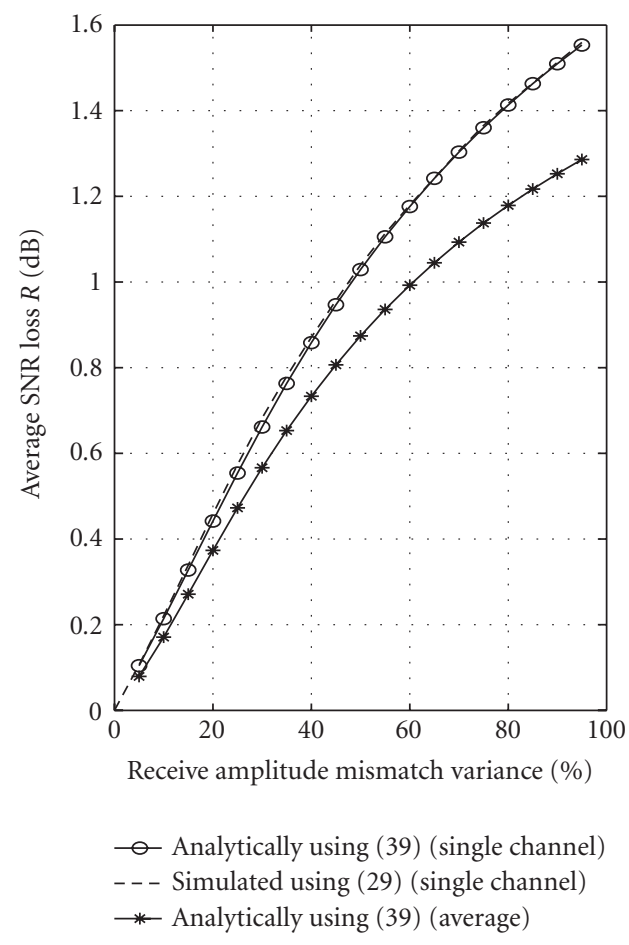

(a)

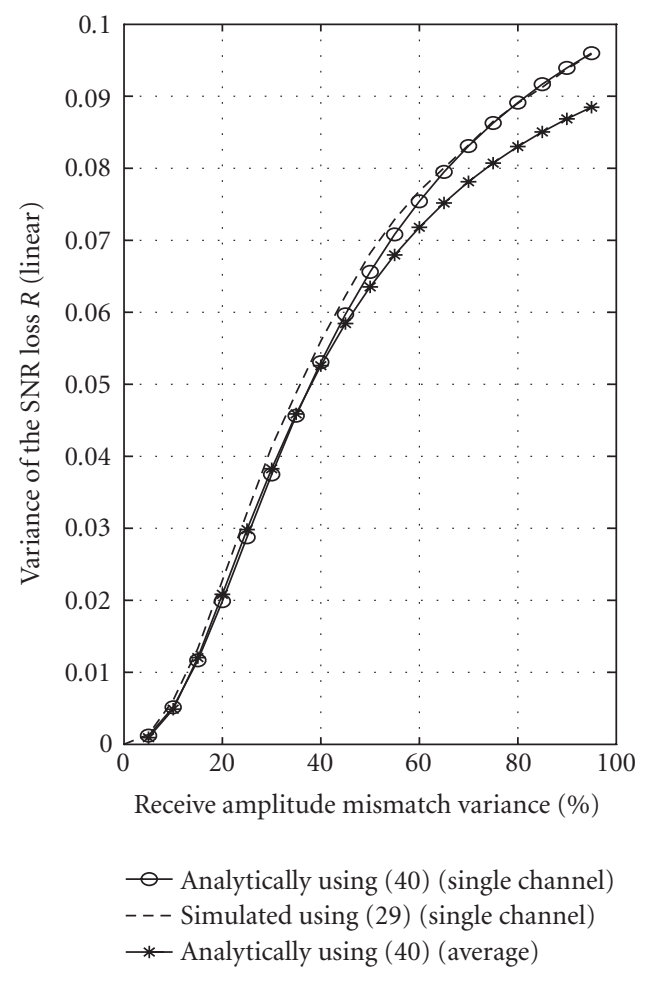

(b)

FIgURE 5: The average (in $\mathrm{dB}$ ) and the variance (in linear units) of the SNR loss $\mathrm{R}$ in presence of receive amplitude mismatches, for $A=2$ transmit antennas.

in which $\phi_{0}=0$. Since $Y$ lies on the $A$-dimensional unit sphere, it is independent of the radius $r$ and is only parametrized by the angles $\left(\phi_{1}, \ldots, \phi_{A-1}\right)$. Therefore, we only need the joint distribution of these angles, which is obtained by the integration of (33) with respect to $r$. For $A=2$, the desired pdf of the single angle $\phi_{1}$ was found to be

$$
\begin{aligned}
& p\left(\phi_{1}\right) \\
& \quad=\frac{c}{2 a}+\frac{c b e^{b^{2} / 4 a} \sqrt{\pi}}{4 a^{3 / 2}}\left[1-\operatorname{erf}\left(-\frac{b}{2 \sqrt{a}}\right)\right], \quad \phi_{1} \in[0,2 \pi] .
\end{aligned}
$$

While the joint distribution of $\left(\phi_{1}, \phi_{2}, \phi_{3}\right)$, for $A=4$, can be shown to be

$$
\begin{aligned}
& p\left(\phi_{1}, \phi_{2}, \phi_{3}\right) \\
& =\frac{c}{16 a^{7 / 2}}\left[2 \sqrt{a}\left(b^{2}+4 a\right)\right. \\
& \left.+b\left(6 a+b^{2}\right) e^{b^{2} / 4 a} \sqrt{\pi}\left(1-\operatorname{erf}\left[-\frac{b}{2 \sqrt{a}}\right]\right)\right], \\
& \phi_{1} \in[0,2 \pi],\left\{\phi_{2}, \phi_{3}\right\} \in[0, \pi] .
\end{aligned}
$$

Finally, reformulating the SNR loss $R$ of (31), in terms of the $A$-dimensional spherical coordinates:

$$
R=\frac{\left(\mu_{A} \cos \phi_{A-1}+\sum_{a=1}^{A-1} \mu_{a} \prod_{k=1}^{A-1} \sin \phi_{k} \cos \phi_{a-1}\right)^{2}}{\sum_{a=1}^{A} \mu_{a}^{2}} .
$$

It is clear that the pdfs of (35) and (36) will enable us to calculate the expected value as well as the variance of $R$, for $A=2$ and $A=4$, respectively, by the evaluation of the following $(A-1)$-tuple finite integrals:

$$
\begin{gathered}
E\{R\}=\int_{0}^{\pi} \cdots \int_{0}^{2 \pi} R\left(\phi_{1}, \ldots, \phi_{A-1}\right) \\
\cdot p\left(\phi_{1}, \ldots, \phi_{A-1}\right) d \phi_{1} \cdots d \phi_{A-1}, \\
\operatorname{Var}\{R\}=\int_{0}^{\pi} \cdots \int_{0}^{2 \pi} R^{2}\left(\phi_{1}, \ldots, \phi_{A-1}\right) \\
\cdot p\left(\phi_{1}, \ldots, \phi_{A-1}\right) d \phi_{1} \cdots d \phi_{A-1} \\
-E^{2}\{R\} .
\end{gathered}
$$

Figure 5 confirms that our analytical characterization is in perfect agreement with the simulated results for the practical system, whose parameters have been highlighted in Section 2.4.

\subsection{Receive phase mismatch only}

This scenario corresponds to the case where the $A$ transmit front-end chains are ideal, and the $A$ receive front-end amplitudes are equal 1 ,

$$
\begin{aligned}
\left\{f_{R x, a}\right\}_{1 \leq a \leq A} & =1, \\
\left\{\left|f_{T x, a}\right|\right\}_{1 \leq a \leq A} & =1 .
\end{aligned}
$$


The SNR loss, $R$, is given by

$$
R=\left|\frac{\sum_{a=1}^{A}\left|h_{a}\right|^{2} e^{-j \arg \left(f_{R x, a}\right)}}{\sum_{a=1}^{A}\left|h_{a}\right|^{2}}\right|^{2} .
$$

Recalling that the phase mismatches are modeled such that $\arg \left(f_{T x}\right)$ and $\arg \left(f_{R x}\right)$ follow the same distribution, which is symmetrical around zero, (40) and (14) are basically equivalent. More importantly, all the results on the characterization of the statistics of linear SNR loss, $R$, obtained for transmit phase mismatch, hold here as well, provided that the value of $\Phi$ is adjusted to that of the receive front ends.

\subsection{Conclusions of impact analysis}

The numerical results of Figures 3, 4, and 5, provided as a validation of our analytical impact analysis, further confirm that the AP multiantenna RF transceivers' amplitude and phase mismatches can severely degrade the transmit MRC performance and may even annihilate most of its potential SNR array gain. The receive amplitude mismatch is shown to be less harmful than its transmit counterpart. This is because it is attenuated by the normalization in the calculation of the transmit MRC weights, as shown in (28). Phase mismatch could cause big SNR loss, since the combining at the receiving user terminal is not in phase any more.

\section{MITIGATION THROUGH MIXED-SIGNAL TRANSCEIVER CALIBRATION}

From Section 3, we have learned that the transmit MRC scheme implies a complex weighting in each of the antenna branches, and that an inaccuracy in these weights caused by multiantenna RF transceivers' amplitude and phase mismatches can lead to a severe performance degradation. The amplitudes and phases of the transmitter and receiver can have widely differing values. Especially, the phases can be totally random due to local oscillator phases, different sampling clock offsets or frequencies, different phase responses of the RF and intermediate frequency (IF) analog filters, different track lengths, different impedance mismatches, and so forth. Although proper and sound design techniques can prevent problems related to local oscillators (LOs) and sampling clocks (common clocks, common LOs), it is much more difficult to guarantee by design that the phases (as well as the amplitudes) of the complete analog chains of all antenna branches are perfectly matched. In order to mitigate this mismatches problem, we advocate a two-step mixedsignal calibration procedure, which, in a first step, measures the frequency responses of the transmitter and receiver antenna branches, and, in as second step, compensates for them digitally.

This section is organized as follows. Section 4.1 determines the calibration requirement to enforce front-end reciprocity. Section 4.2 discusses the most important calibration methods that have been proposed in the state of the art. Section 4.3 explains our proposed calibration method in more detail, whereas Section 4.4 describes its practical implementation and integration into a real-time wireless MIMO-OFDM prototyping platform.

\subsection{Front-end reciprocity requirement}

We can notice from (5), that, for a maximum SNR TX-MRC solution, the multiantenna transceiver (TRX) has to fulfill

$$
f_{T x, a}=\xi f_{R x, a}, \quad 1 \leq a \leq A,
$$

where $\xi$ is a complex scalar. This requirement can be reformulated into

$$
\frac{f_{T x, a}}{f_{R x, a}}=\xi, \quad 1 \leq a \leq A,
$$

which can be interpreted as a matching problem. Because of the unpredictable characteristics of analog TRX FEs, a method to digitally calibrate the gain and phase mismatch is desirable,

$$
c_{a} \cdot \frac{f_{T x, a}}{f_{R x, a}}=\alpha, \quad 1 \leq a \leq A,
$$

where the $c_{a}$ 's are the complex calibration scalars to be identified, and $\alpha$ is another complex scalar.

\subsection{State-of-the-art calibration methods}

To the authors' knowledge, four different types of amplitude and phase mismatch calibration methods can be identified in the state of the art.

The first method, which performs separate TX and RX gain and phase mismatch calibration, was proposed in $[7,9$, $10]$. In this method, the absolute value of both the transmitter and receiver frequency responses needs to be estimated before the calibration can be done, which requires a veryhigh RF circuit complexity, mainly caused by the need for a very accurate and, hence, expensive RF signal generator.

The second method, which relies on the normalized least mean squares (NLMS) algorithm, was proposed in [8]. Unfortunately, this multiantenna receiver mismatch calibration scheme only works at the receive side, whereas TX-MRC processing requires calibration at the transmit side.

The third method, which is an essential part of the Qualcomm IEEE802.11n proposal, has been proposed in [17]. This, so-called "over-the-air" calibration method, first measures the composite channels (propagation channel, including analog TRXs) in both the up- and downlink direction, and signals the measurement obtained at one side of the link to the other side. The knowledge of the measured upand downlink channels at each side, finally, allows to enforce channel reciprocity in both the AP and the terminal. One major problem with this method is that the calibration needs to be completely redone, once the user terminal setup changes, for example, when a user terminal is switched off and on again, or, when a new user terminal joins the communication setup.

As we will show in Section 4.3, our mixed-signal calibration method avoids the need for knowledge about the absolute value of the TRX frequency responses, such that the 


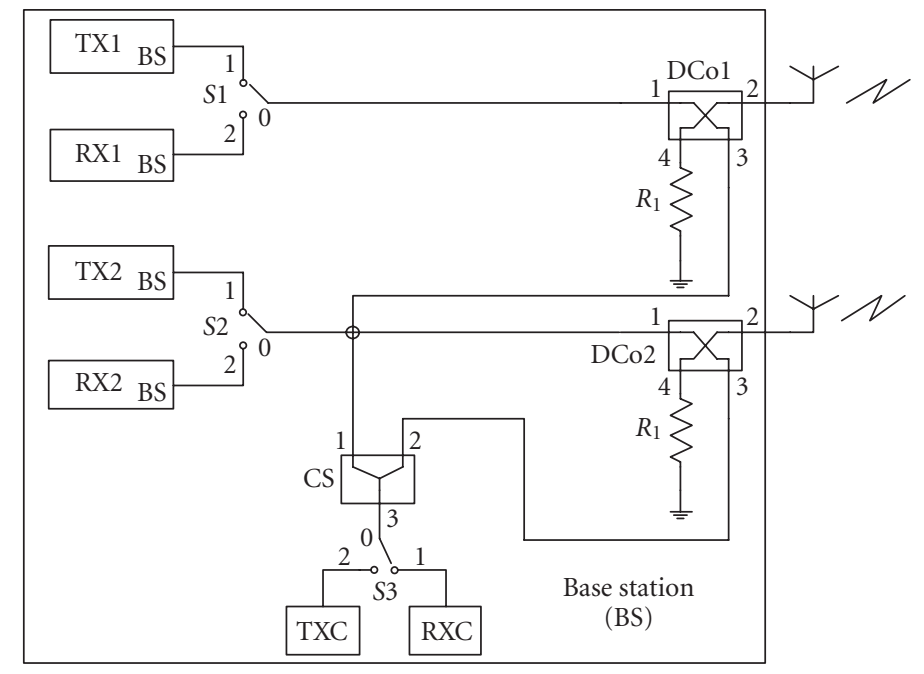

FIGURE 6: Structure of the proposed calibration loop.

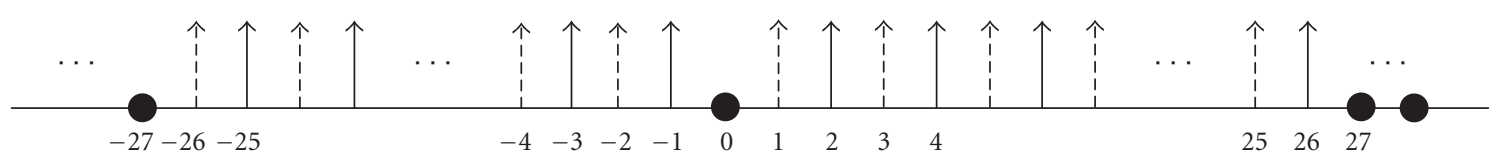

FIGURE 7: Position of the subcarriers with signal; solid arrows for TX1, dashed arrows for TX2, and black dots for zero subcarriers.

RF circuit complexity can be significantly reduced. Furthermore, it does not involve the user terminal, hence, avoiding the need for time-consuming recalibration upon terminal change. In fact, our calibration method is quite stable, only requiring recalibration every few hours.

\subsection{Proposed calibration method}

We propose, in a first step, to use a calibration transceiver (TRX) to measure the frequency response of the AP's TRX FEs, and, in a second step, to calibrate the amplitude and phase mismatch digitally, as shown in Figures 6 and 8. In Figure 6, TX1, TX2 and RX1, RX2 are two transmitters and two receivers of the AP for a multiantenna system. TXC and $\mathrm{RXC}$ are the calibration transmitter and receiver, also implemented in the AP. S1, S2, and S3 are three switches for TDD operation; DCo1 and DCo2 are two power directional couplers; and CS is a power combiner/splitter.

The signals transmitted during calibration are dedicated test signals, typically training symbols with low peak-toaverage power ratio (PAPR). To ensure that RXC can distinguish between the test signals coming from TX1 and TX2, TX1 and TX2 transmit on interleaved subsets of the 52 OFDM data subcarriers, as shown in Figure 7. Therefore, TX1 and TX2 can transmit their signals simultaneously. The signals coming from TX1 and TX2 will pass through directional couplers, and will be combined at the power combiner before being received by RXC. TXC transmits training symbols on all the 52 OFDM data subcarriers. The signal will undergo the power splitter and the two directional couplers to RX1 and RX2. The test signals are BPSK signals; channel estimation is performed at the receivers, so that the final received signals are the TFs corresponding to that transmission chain.

From the calibration loop, we can measure four transfer functions as shown in (44). As TF1 and TF2, each have information on 26 subcarriers (interleaved), curve fitting in the frequency domain must be applied to recover the missing information. Curve fitting is implemented by linear interpolation in band and holding at the edges of the band. After the curve fitting, all the 4 TFs have information on 52 subcarriers, division TF3/TF1 and TF4/TF2 can be done.

$$
\begin{aligned}
& T F 1=f_{T X, 1} \cdot f_{S 1_{\text {_port } 1 \rightarrow \operatorname{port} 0} \cdot f_{D C o 1} \text { port } 1 \rightarrow \text { port } 3} \\
& \text { - } f_{C S_{-} \text {port } 1 \rightarrow \text { port } 3} \cdot f_{S 3_{\text {_ port }} \rightarrow \text { port } 1} \cdot f_{R X C}, \\
& T F 2=f_{T X, 2} \cdot f_{S 2 \text { port } 1 \rightarrow \text { port } 0} \cdot f_{D C o 2 \text { port } 1 \rightarrow \text { port } 3} \\
& \text { - } f_{C S_{\text {_ port } 2 \rightarrow \text { port } 3}} \cdot f_{S 3_{\text {_ port }} \rightarrow \operatorname{port} 1} \cdot f_{R X C}, \\
& T F 3=f_{T X C} \cdot f_{S 3 \text { port } 2 \rightarrow \text { port } 0} \cdot f_{C S_{\text {_ port } 3 \rightarrow \text { port } 1}} \\
& \text { - } f_{D C o 1_{\text {p port } 3 \rightarrow \text { port } 1}} \cdot f_{S 1_{\text {p port } 0 \rightarrow \operatorname{port} 2}} \cdot f_{R X, 1},
\end{aligned}
$$

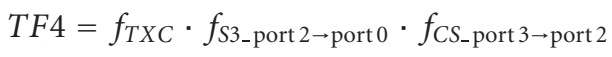

$$
\begin{aligned}
& \text { - } f_{D C o 2 \text { port } 3 \rightarrow \text { port } 1} \cdot f_{S 2 \text { port } 0 \rightarrow \text { port 2 }} \cdot f_{R X, 2} \text {. }
\end{aligned}
$$

Due to the reciprocity of the power devices and the nonreciprocity of the switches, we obtain 


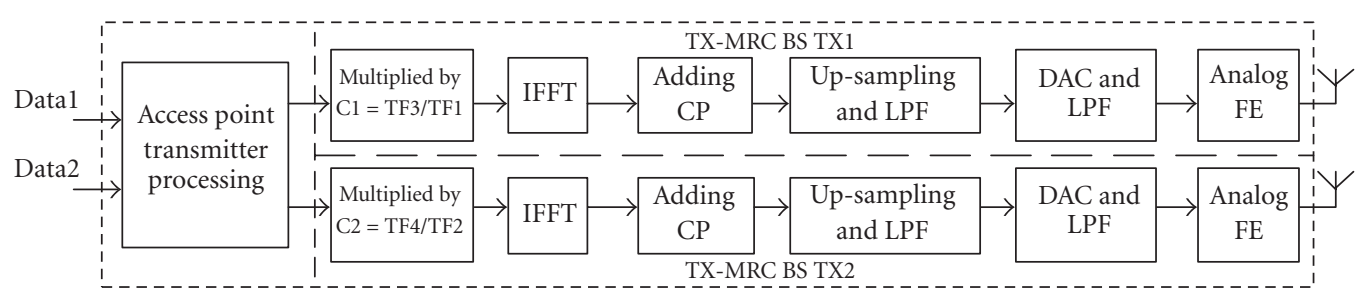

FIGURE 8: Implementation position of the calibration factors.

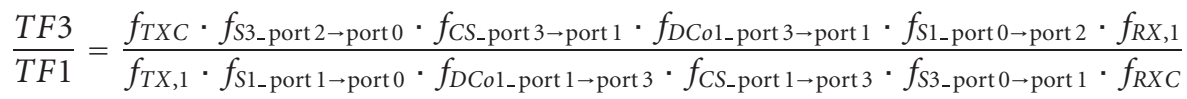

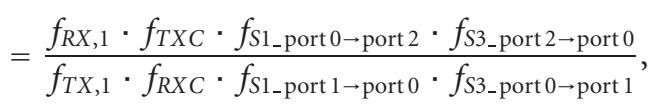

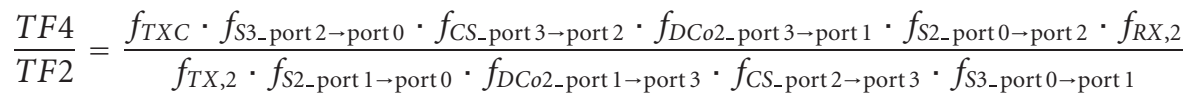

$$
\begin{aligned}
& =\frac{f_{R X, 2} \cdot f_{T X C} \cdot f_{S 2 \_ \text {port } 0 \rightarrow \text { port } 2} \cdot f_{S 3_{-} \text {port } 2 \rightarrow \text { port } 0}}{f_{T X, 2} \cdot f_{R X C} \cdot f_{S 2 \text { port } 1 \rightarrow \text { port } 0} \cdot f_{S 3_{-} \text {port } 0 \rightarrow \text { port } 1}} .
\end{aligned}
$$

In the result of TF3/TF1 and TF4/TF2, the effect of directional coupler and splitter/combiner is cancelled due to their reciprocity. The switches S1 and S2 are not reciprocal, but their nonreciprocity is part of what needs to be calibrated, so $\left(S 1_{\text {_ }}\right.$ port $\left.0 \rightarrow \operatorname{port} 2\right) /\left(S 1_{\text {_ }}\right.$ port $\left.1 \rightarrow \operatorname{port} 0\right)$ can be absorbed into $f_{R X, 1} / f_{T X, 1}$; similarly, $\left(S 2_{-}\right.$port $0 \rightarrow$ port 2$) /(S 2$ - port $1 \rightarrow$ port 0$)$ can be absorbed into $f_{R X, 2} /$ $f_{T X, 2}$, leading to

$$
\begin{aligned}
& \frac{T F 3}{T F 1}=\frac{f_{R X, 1}}{f_{T X, 1}} \cdot \alpha=c_{1}, \\
& \frac{T F 4}{T F 2}=\frac{f_{R X, 2}}{f_{T X, 2}} \cdot \alpha=c_{2} .
\end{aligned}
$$

Here, $\alpha$ is a common factor, defined as

$$
\alpha=\frac{f_{T X C} \cdot f_{S 3 \_ \text {port } 2 \rightarrow \text { port } 0}}{f_{R X C} \cdot f_{S 3_{-} \text {port } 0 \rightarrow \text { port } 1}} .
$$

Therefore, the calibrating matrix can be written as

$$
\mathbf{C}=\left[\begin{array}{cc}
c_{1} & 0 \\
0 & c_{2}
\end{array}\right]
$$

In the implementation, the positions of $c_{1}$ and $c_{2}$ are shown in Figure 8. By multiplying $c_{1}$ and $c_{2}$ into the TX blocks, the reciprocity requirement in (43) is achieved.

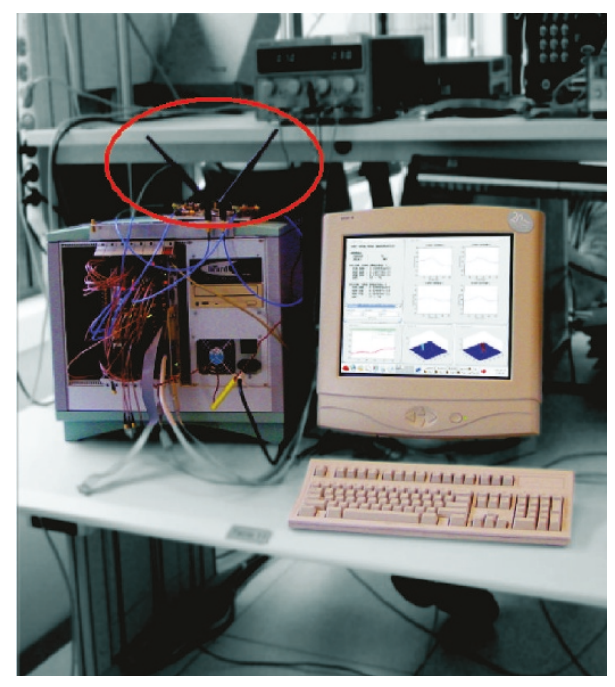

FIGURE 9: PICARD: IMEC's MIMO-OFDM prototyping platform.

\subsection{Implementation and measurement results}

The proposed calibration method has been implemented in IMEC's MIMO prototyping platform, the so-called PICARD platform (see Figure 9). This platform supports realtime wireless MIMO transmission schemes with either transmit or receive processing. The calibration procedure with an auxiliary front end, as described in Section 4.3, has been 


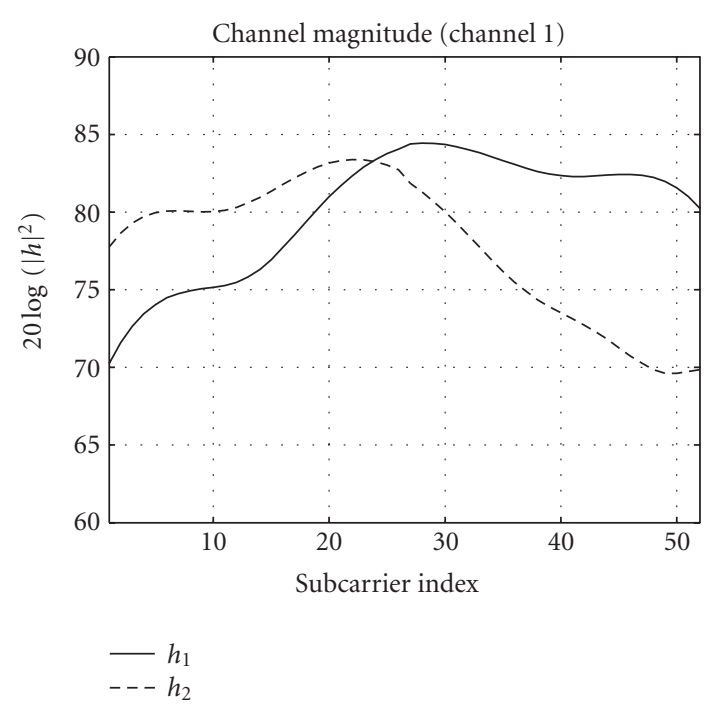

(a)

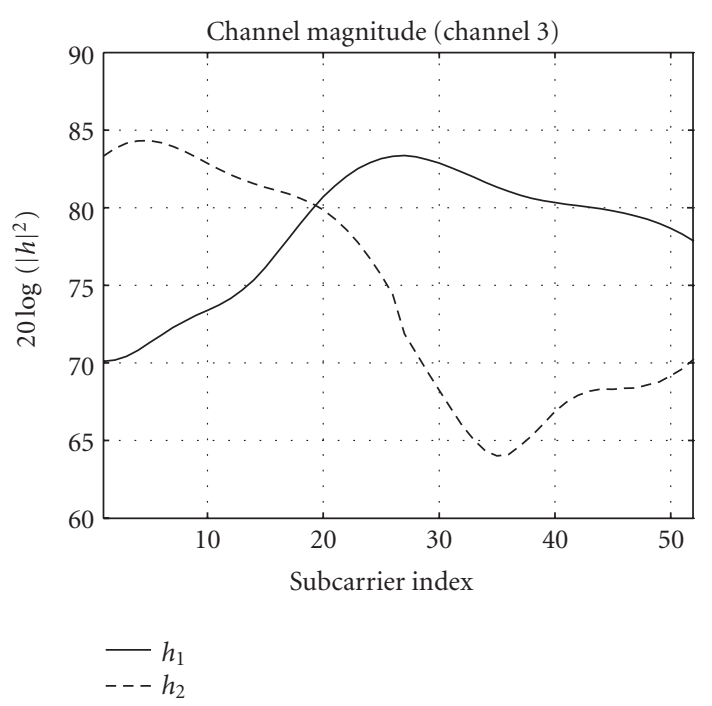

(c)

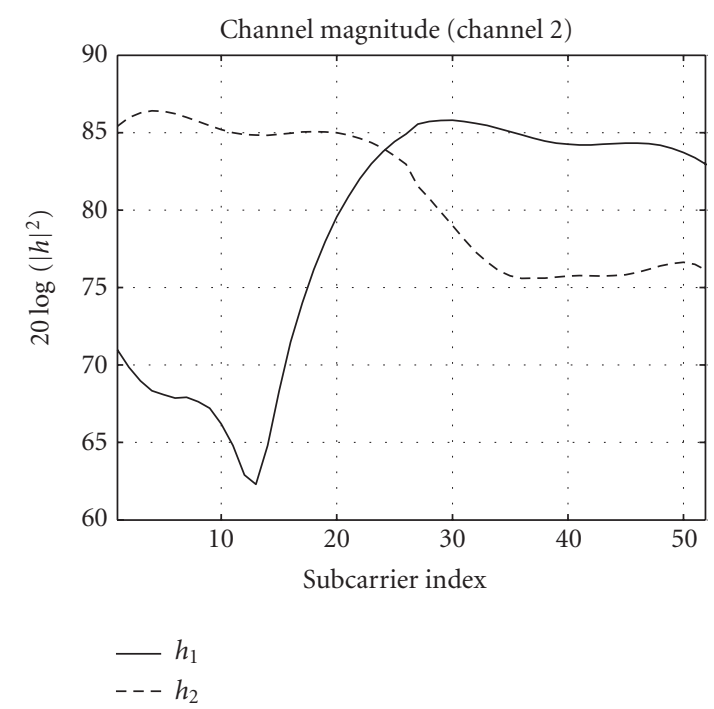

(b)

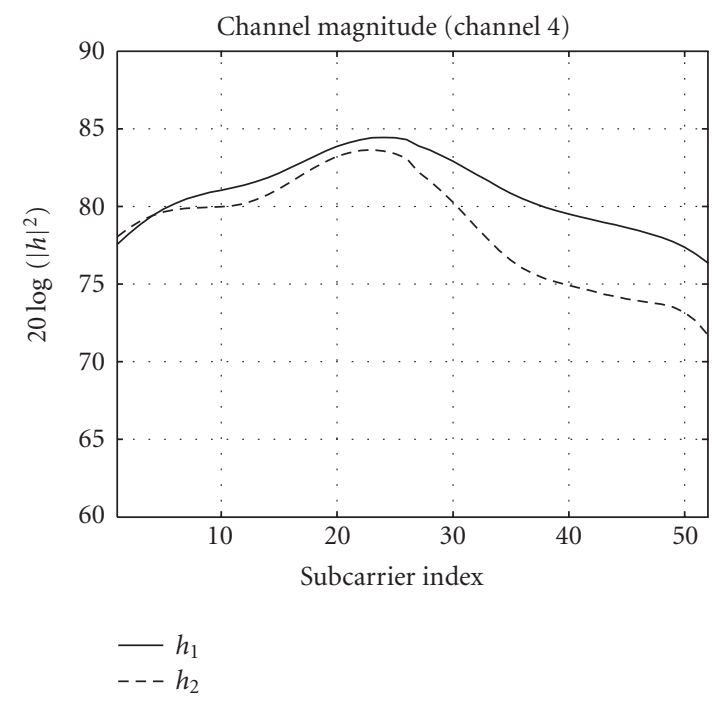

(d)

FIgURe 10: Magnitude of the channel frequency responses.

integrated into this platform. In this way, transmit processing with reverse link channel estimation is possible. The maximum number of antennas is two at either side. From this paper's perspective, a " $2 \mathrm{x} 1$ " TX-MRC system can be tested. In this setup, the receiver is a conventional single-antenna receiver. When TX-MRC is used, the platform is configured with the PHY layer parameters (modulation format, preambles, etc.) of the IEEE802.11a/g standard [14].

We have carried out wireless measurements at $5 \mathrm{GHz}$ in the RF-DSP lab of IMEC over various indoor propagation channels. We present here the results over four representative channels. Channels 1 to 3 featured significant multipath (no direct line of sight), whereas channel 4 had a direct line-of-sight component. The channel frequency responses are shown in Figure 10. Note that the room had moderate dimensions, which explains that there are no sharp dips in the channel frequency responses.

For each channel, ten SNR measurements were made. The SNR was obtained by the error vector magnitude (EVM) of the received constellations. The EVM measures the mean of the magnitude of the error of the constellation points. For normalized constellations, the inverse of this mean value is a good estimate of the postprocessing SNR, provided that the constellation clouds do not overlap too much.

Table 1 shows the mean and variance of the SNR measured over the four channels, with and without calibration. When calibrated front ends are used, an improvement on the mean SNR of 1 to $4 \mathrm{~dB}$ is observed. The observed SNRs fluctuate, which are due to several reasons: data dependency of the constellation symbols, noise fluctuations, and channel 
TABLE 1: Measured SNRs with TX-MRC over 4 indoor channels.

\begin{tabular}{lcccc}
\hline & \multicolumn{2}{c}{ No calibration } & \multicolumn{2}{c}{ With calibration } \\
\hline & Mean SNR & Var SNR & Mean SNR & Var SNR \\
\hline Chan 1 & 15.50 & 0.34 & 18.19 & 1.05 \\
Chan 2 & 15.42 & 0.50 & 17.48 & 2.89 \\
Chan 3 & 13.14 & 0.55 & 16.80 & 1.30 \\
Chan 4 & 18.49 & 0.26 & 19.48 & 1.81 \\
\hline
\end{tabular}

time variation. Of these three, the channel time variations have the highest impact. Indeed, there is a time delay of about $10 \mathrm{~ms}$ in our setup between the reverse link channel estimation and the actual use of the channel in the forward link, so that the channel estimate is sometimes outdated. Therefore, channel time variation can have some adverse impact on the performance improvement of the transmit MRC. Finally, we notice that the variance of the SNR is higher with calibration than without calibration. This can be related again to channel variations. When the calibration is not applied, we are in a situation where the MRC weights are not optimal, whether or not the channel is outdated, and the effect of channel variation is not very visible. On the contrary, with calibration, the highest SNR is obtained when the channel is static, and significant SNR reduction occurs when the channel is not static. Hence, higher SNR variations are observed in the calibrated case due to channel variations.

Globally, as can be seen from the mean SNRs, the calibrated front ends perform significantly better over a real wireless channel, as could be expected from the analysis and performance results in Section 3.

\section{CONCLUSION}

In this paper, we have presented a novel statistical analysis to assess the impact of multiantenna RF transceivers' amplitude and phase mismatches on a transmit MRC system that relies on the CSI estimate obtained during the uplink phase to determine the transmit MRC weights employed during the downlink phase. The obtained numerical results, for a MISO-OFDM system operating over realistic spaceand frequency-selective indoor channels, suggest that these effects can completely annihilate the SNR gain promised by an ideal transmit MRC system. More importantly, the receive amplitude mismatch seems to be less harmful than its transmit counterpart, since the former is attenuated by a normalization factor that appears in the calculation of the transmit MRC weights. Furthermore, phase mismatch could induce big performance degradation, due to the fact that the signals at the receiving user terminal combine out of phase instead of in phase.

To mitigate the detrimental effect of multiantenna RF transceivers' amplitude and phase mismatches in transmit MRC, we have proposed a novel mixed-signal calibration scheme, which, first, measures, via additional RF calibration hardware, the actual multiantenna transmit and receive front-end mismatches, and, subsequently, compensates for these mismatches in the digital domain. Simulation results indicate that the proposed calibration scheme can reduce the implementation loss to as little as a few tenths of a dB. Furthermore, our scheme exhibits two major advantages compared to competing alternatives described in the state of the art. First, it avoids the need for knowledge about the $a b$ solute value of the transceivers' frequency responses, hence, significantly reducing the complexity of the additional RF calibration hardware. Second, it does not involve the user terminal in the calibration process, hence, avoiding the need for a time- and bandwidth-consuming recalibration procedure upon changes in the user terminal setup. Last but not least, to demonstrate the feasibility of our calibration scheme in real life, we have described its practical implementation and integration into a real-time wireless MIMO-OFDM prototyping platform.

\section{REFERENCES}

[1] “IEEE 802.11 homepage," http://www.ieee802.org/11.

[2] D. Gesbert, M. Shafi, D.-S. Shiu, P. J. Smith, and A. Naguib, "From theory to practice: an overview of MIMO space-time coded wireless systems," IEEE Journal on Selected Areas in Communications, vol. 21, no. 3, pp. 281-302, 2003.

[3] A. J. Paularj, D. A. Gore, R. U. Nabar, and H. Bolcskei, "An overview of MIMO communications - a key to gigabit wireless," Proceedings of IEEE, vol. 92, no. 2, pp. 198-218, 2004.

[4] G. L. Stuber, J. R. Barry, S. W. McLaughlin, Y. Li, M. A. Ingram, and T. G. Pratt, "Broadband MIMO-OFDM wireless communications," Proceedings of IEEE, vol. 92, no. 2, pp. 271-294, 2004.

[5] T. K. Y. Lo, "Maximum ratio transmission," IEEE Transactions on Communications, vol. 47, no. 10, pp. 1458-1461, 1999.

[6] A. J. Paulraj, R. U. Nabar, and D. A. Gore, Introduction to Space-Time Wireless Communications, Cambridge University Press, Cambridge, UK, 2003.

[7] R. B. Ertel, Z. Hu, and J. H. Reed, "Antenna array hardware amplitude and phase compensation using baseband antenna array outputs," in Proceedings of 49th IEEE Vehicular Technology Conference (VTC'99), vol. 3, pp. 1759-1763, Houston, Tex, USA, May 1999.

[8] S. Kobayakawa, M. Tsutsui, and Y. Tanaka, "A blind calibration method for an adaptive array antenna in DS-CDMA systems using an MMSE algorithm," in Proceedings of 51st IEEE Vehicular Technology Conference (VTC'00), vol. 1, pp. 21-25, Tokyo, Japan, May 2000.

[9] H. G. Park, C. Park, H. Oh, and M. G. Kyeong, "RF gain/phase and I/Q imbalance error correction technique for multichannel array antenna systems," in Proceedings of 53rd IEEE Vehicular Technology Conference (VTC '01), vol. 1, pp. 175179, Rhodes, Greece, May 2001.

[10] J. H. Jung, H. G. Park, and D. S. Ryu, "Calibration techniques of multi-channel transceiver using noninterfering calibration signals for CDMA smart antenna systems," in Proceedings of 6th IEEE International Conference on Signal Processing (ICSP '02), vol. 2, pp. 1629-1632, Beijing, China, August 2002 .

[11] A. Bourdoux, B. Come, and N. Khaled, "Non-reciprocal transceivers in OFDM/SDMA systems: impact and mitigation," in Proceedings of IEEE Radio and Wireless Conference (RAWCON '03), pp. 183-186, Boston, Mass, USA, August 2003.

[12] N. Khaled, S. Jagannathan, F. Petré, G. Leus, and H. De Man, "On the impact of multi-antenna RF transceivers' amplitude 
and phase mismatches on transmit MRC," in Proceedings of IEEE International Conference on Acoustics, Speech, and Signal Processing (ICASSP '05), vol. 4, pp. 893-896, Philadelphia, Pa, USA, March 2005.

[13] J. Liu, A. Bourdoux, J. Craninckx, et al., "OFDM-MIMO WLAN AP front-end gain and phase mismatch calibration," in Proceedings of IEEE Radio and Wireless Conference (RAWCON '04), pp. 151-154, Atlanta, Ga, USA, September 2004.

[14] IEEE Std. 802.11a, "Wireless LAN Medium Access Control (MAC) and Physical Layer (PHY) specifications: high-level physical in the $5 \mathrm{GHz}$ band," Tech. Rep., IEEE, New York, NY, USA, September, 1999.

[15] V. Erceg, L. Schumacher, P. Kyritsi, et al., "TGn channel models," IEEE 802.11 document 03/940r2, IEEE, New York, NY, USA, January 2004.

[16] J. G. Proakis, Digital Communications, McGraw-Hill, New York, NY, USA, 3rd edition, 1995.

[17] J. Ketchum, S. Howard, M. Wallace, R. Walton, B. Bjerke, I. Medvedev, et al., "PHY design for spatial multiplexing MIMO WLAN," IEEE 802.11 document 04/0721r0, IEEE, New York, NY, USA, July 2004.

Jian Liu received her M.S. and B.S. degrees in electrical engineering from Nankai University, Tianjin, China, in 1998 and 1995, respectively. From 1998 to 2001, she was working as an Activity Leader on wireless local loop (WLL) technology in Great Dragon Telecom., Beijing, China. In October 2001, she joined the wireless research group of IMEC in Leuven, Belgium, working towards a Ph.D. degree. Her research in-

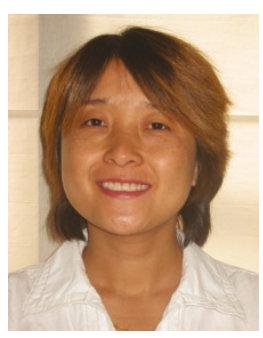
terests are in the impact and mitigation of analog front-end impairments in multiple-antenna MIMO wireless communication systems.

Nadia Khaled received the M.S. degree in electrical engineering from L'Ecole Nationale Supérieure d'Electrotechnique, d'Electronique, d'Informatique, d'Hydraulique et des Télécommunications (ENSEEIHT), Toulouse, France, in 2000. Since the completion of the Katholieke Universiteit Leuven predoctoral examination in May 2001, she has been pursuing her Ph.D. research with the wireless research group of IMEC,

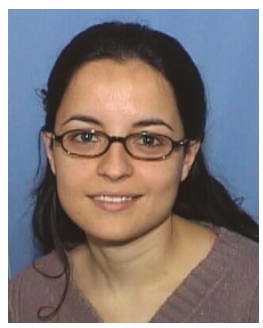
Leuven, Belgium, as a Ph.D. student at the Katholieke Universiteit, Leuven. Her research interests lie in the area of signal processing for wireless communications, particularly MIMO techniques and transmit optimization schemes.

Frederik Petré is a Senior Project Engineer at the Flanders' MECHATRONICS Technology Center (FMTC), which is a new research centre, operating since October 2003, with the mission to establish a bridge between the academic and industrial knowhow in mechatronics in Flanders, Belgium. Over there, he focuses on end-to-end system design and integration of a mobile wireless sensor system for machine diag-

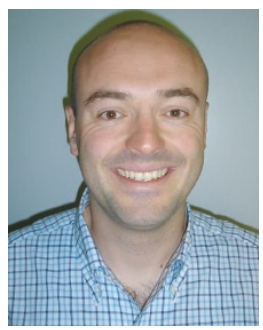
nosis within the very relevant industrial process control application context. Before joining FMTC, Frederik was a Senior Scientist within the Wireless Research Group at the Interuniversity Microelectronics Centre (IMEC), investigating baseband signal processing algorithms and digital architectures for future generation wireless communication systems, including third-generation (3G) and fourth-generation $(4 \mathrm{G})$ broadband cellular networks and highthroughput wireless local area networks (HT-WLANs). He received the M.S. (1997) and the Ph.D. (2003) degrees in electrical engineering, both from the Katholieke Universiteit Leuven, Belgium. During the fall of 1998, he spent 6 weeks as a Visiting Researcher at the Information Systems Laboratory (ISL), Stanford University, Calif, USA, working on OFDM-based powerline communications. Frederik is a Member of the ProRISC Technical Program Committee and Secretary of the IEEE Benelux Section on Communications and Vehicular Technology (CVT). In 2005, he served as a Guest Editor for the EURASIP Journal on Wireless Communications and Networking (JWCN), resulting in a special issue on Reconfigurable Radio for Future Generation Wireless Systems. From January 2004 till December 2005, he was a Member of the Executive Board of the European 6th Framework Network of Excellence in Wireless Communications (NEWCOM), and the Leader of NEWCOM Project D on Flexible Radio.

André Bourdoux received the M.S. degree in electrical engineering (specialisation in microelectronics) in 1982 from the Université Catholique de Louvain-la-Neuve, Belgium. He is coordinating the research on multiantenna communications in the wireless research group at IMEC. His current interests span the areas of wireless communications theory, signal processing, and transceiver architectures with a special em-

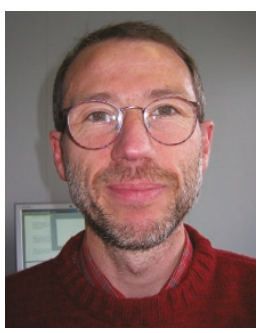
phasis on broadband and multiantenna systems. Before joining IMEC, his research activities were in the field of algorithms and RF architectures for coherent and high-resolution radar systems. He is the author and coauthor of several conference and journal papers and of two patents applications in the field of SDMA and MIMO transmission.

Alain Barel was born in Roeselare, Belgium, on July 27, 1946. He received the degree in electrical engineering from the Université Libre de Bruxelles, Belgium in 1969, the postgraduate degree in telecommunications from Rijks Universiteit Gent (State University of Ghent), Belgium in 1974, and became Doctor of Applied Science from the Vrije Universiteit Brussel (Free University of Brussels) in 1976. He worked as Assistant

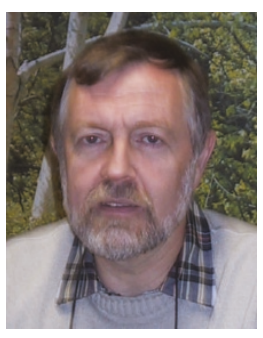
and Lecturer at the Vrije Universiteit Brussel. Currently, he is a full Professor in the Department of Fundamental Electricity and Instrumentation at the same university, and teaches electromagnetism and microwaves. His research interests lie in the modeling and measuring of nonlinear microwave telecommunication components. 